\title{
Impact of admission blood glucose level on outcomes in community-acquired pneumonia in older adults
}

This article was published in the following Dove Press journal:

International Journal of General Medicine

3 May 2013

Number of times this article has been viewed

\section{Rajib K Bhattacharya Jonathan D Mahnken Sally K Rigler}

University of Kansas School of Medicine, Kansas City, KS, USA
Correspondence: Rajib K Bhattacharya University of Kansas School of Medicine, 390I Rainbow Boulevard, Kansas City, KS 66160, USA

Tel + I $9 \mid 35886022$

Fax + I 9135884060

Email rbhattacharya@kumc.edu
Background: Community-acquired pneumonia (CAP) is a common cause of morbidity and mortality in older adults. Although diabetes mellitus is a risk factor for pneumonia, the clinical impact of blood glucose level at the time of admission is not clear. Our goal was to examine the association between admission hyperglycemia and subsequent mortality, length of stay, and readmission outcomes in older adults with CAP.

Methods: A retrospective observational study was conducted using hospital data for communityacquired pneumonia admissions in 857 persons from January 1, 2008 to December 31, 2010. We examined the effects of admission glucose level on mortality, length of stay, and 30 day readmission, adjusted for demographic factors and comorbidity.

Results: The mean age of the sample was 64 years, and $51 \%$ of the subjects were female. Inpatient mortality occurred in $4.6 \%$ and the median length of stay was 5 days (interquartile range 3-9 days). Readmission within 30 days occurred in 17\%. We found little impact of first glucose measures on in-hospital mortality $(P=0.94)$, length of stay $(P=0.95)$, and 30 -day readmission $(P=0.56)$. Subjects 65 years and older trended towards higher in-hospital mortality. Older age, cancer, heart failure, and cirrhosis were associated with adverse outcomes.

Conclusion: Glucose level upon admission for community-acquired pneumonia was not associated with adverse outcomes within 30 days in older adults.

Keywords: community-acquired pneumonia, hyperglycemia, readmission rates, hospital mortality

\section{Introduction}

Pneumonia is the eighth most common cause of death in the USA and ranks second among infectious causes of mortality. ${ }^{1,2}$ Over five million people develop pneumonia every year in the US, mostly older adults. ${ }^{3}$ Diabetes mellitus is a risk factor for pneumonia, and as many as one-quarter of persons with community-acquired pneumonia (CAP) are diabetic. ${ }^{3,4}$ Hyperglycemia has been established as an independent predictor of morbidity and mortality in patients admitted with acute coronary syndromes, ischemic stroke, trauma, heart failure, and coronary artery bypass graft (CABG), as well as admission to surgical and medical intensive care units. ${ }^{5,6}$ Although hyperglycemia leads to impaired host immune responses, the impact of hyperglycemia on concrete clinical outcomes (eg, mortality, length of stay, and readmission) is not clear in CAP. Data on hyperglycemia and CAP are limited. The existing relevant literature shows mixed results, with most studies focused on hospital complications associated with hyperglycemia. ${ }^{4-8}$

Glycemic status at admission is readily available for all patients, and might provide a potentially useful predictive factor for outcomes and utilization of care. Our goal was to 
examine potential associations of admission hyperglycemia with mortality, length of stay, and readmission rates in patients with CAP.

\section{Methods}

\section{Cohort and measures}

Retrospective observational data were extracted from admissions to the University of Kansas Medical Center for community-acquired pneumonia (ICD-9-CM code 480-487) from January 1, 2008 through December 31, 2010. Only persons aged 40 years and older were sampled. Our analysis examines the effects of first glucose measure upon admission and age on hospital mortality, length of stay (LoS), and readmission for community-acquired pneumonia (CAP) within 30 days. Data for a total of 1012 CAP admissions were provided. In addition to the primary explanatory measures, demographics (sex and race/ethnicity [American Indian/ Eskimo/Aleutian, black, Hispanic, Oriental/Asian/Pacific Islander/Indonesian, white, or other]) were also analyzed. Medical comorbidities, including the presence or absence of cancer, chronic heart failure, chronic renal failure, cirrhosis, HIV, and diabetes were provided in the hospital discharge data. We assigned the patients to three groups. Glucose was categorized as normal glycemia $(<126)$, mild hyperglycemia (126-<180), and uncontrolled hyperglycemia $(\geq 180)$.

\section{Statistical analysis}

Univariate descriptive statistics (mean, standard deviation, and percentiles for continuous measures and frequencies and relative frequencies for categorical measures) were derived. Bivariate analyses were also conducted by generating contingency tables with glucose $(<126 / 126$ to $<180 / \geq 180)$, and Pearson's Chi-square test or exact test alternatives were conducted. Descriptive statistics for age within glucose levels were also generated, and age distributions across these groups was performed using one-way analysis of variance. Comparisons of LoS between strata were performed using the log-rank test. Unconditional logistic regression was used to model in-hospital mortality and 30-day hospital readmission as a function of glucose and age, adjusted for other potential confounding variables such as sex, race/ethnicity, and comorbid conditions. (Diabetes was not included as a covariate due to its strong association with the explanatory measure of interest: glucose.) Models were built using stepwise selection with an entry criterion of a score test $P$-value less than 0.15 and a retaining criteria of $P<0.10$ from the Wald test. An interaction term between glucose and age group was also evaluated in a model that was adjusted for the other covariates selected from this model building process. These enabled the assessment of whether the effects of glucose on mortality and on readmission were different among those ages 65 and older compared to those ages 40-64. The final models were assessed using the Hosmer-Lemeshow goodness-of-fit test. Adjusted odds ratios and corresponding 95\% Wald confidence intervals (CI) were generated. Cox proportional hazards regression was used to model LoS as a function of glucose and age, adjusted for other potential confounding variables such as sex, race/ ethnicity, and comorbid conditions. Subjects who died; were discharged to hospice; transferred to another institution; or left against medical advice, were treated as right-censored observations for this analysis. A model was built using the same stepwise procedure as described above for the logistic regression models, and analogous testing for an interaction effect between glucose category and age group was conducted. Adjusted hazard ratios and corresponding 95\% Wald CI were generated.

All data management and data analysis were performed using SAS version 9.2 (2002-2008, SAS Institute Inc, Cary, $\mathrm{NC}, \mathrm{USA}$ ).

\section{Results}

One thousand and twelve community-acquired pneumonia admissions were identified. Missing data required exclusion of 21 records (one hospitalization was missing a valid code for disposition; three records were missing data for the race variable; and 17 were missing a glucose measure for the corresponding admission). After these exclusions, 991 hospitalization records remained which were generated by 857 unique subjects because of repeated hospitalizations in some individuals. Each of these was allowed to contribute only one hospitalization to the analysis. Thus, these 857 subjects comprised the sample for the mortality and length of stay outcome measures (Table 1). However, for the analysis of readmission rates, we further excluded those who died in the hospital or were discharged to hospice, leaving a final sample of 797 persons (Table 2).

The mean age of the sample was 64 years, and 51\% were female. Over $70 \%$ of the population was non-Hispanic white. Comorbidities are shown in Table 1. Glucose was categorized as normoglycemia in 58.1\%, mild hyperglycemia in $27.8 \%$, and uncontrolled hyperglycemia in $14 \%$. Twenty-nine percent had a recognized diagnosis of diabetes mellitus; this diagnosis was associated with higher glucose levels $(P<0.001)$.

In Table 1, inpatient mortality occurred in $4.6 \%$. The median length of stay was 5 days, (inter-quartile 
Table I Baseline characteristics of all the patients admitted for pneumonia

\begin{tabular}{|c|c|c|c|c|c|}
\hline & All subjects** & Normal & $\begin{array}{l}\text { Mild } \\
\text { hyperglycemia }\end{array}$ & $\begin{array}{l}\text { Uncontrolled } \\
\text { hyperglycemia }\end{array}$ & $P$-value**** \\
\hline & & $<126 \mathrm{mg} / \mathrm{dl}$ & $126-<180 \mathrm{mg} / \mathrm{dl}$ & $\geq 180 \mathrm{mg} / \mathrm{dl}$ & \\
\hline Total & 857 & 498 & 238 & $|2|$ & \\
\hline \multicolumn{6}{|l|}{ Age } \\
\hline Years* & $64.1 \pm 13.3$ & $63.3 \pm 13.7$ & $65.4 \pm 13.0$ & $64.8 \pm 12.2$ & 0.121 \\
\hline $65+$ & $401(46.8)$ & $227(45.6)$ & II 5 (48.3) & $59(48.8)$ & 0.703 \\
\hline Male & $416(48.5)$ & $234(47.0)$ & $122(5 \mid .3)$ & $60(49.6)$ & 0.538 \\
\hline Heart failure & $183(2 \mid .4)$ & $90(18.1)$ & $61(25.6)$ & $32(26.5)$ & 0.022 \\
\hline Cirrhosis & $22(2.57)$ & $14(2.8 \mathrm{I})$ & $3(1.26)$ & $5(4.13)$ & 0.231 \\
\hline Chronic renal failure & $161(18.8)$ & $83(16.7)$ & $50(21.0)$ & $28(23.1)$ & 0.154 \\
\hline HIV & $4(0.47)$ & $4(0.80)$ & 0 & 0 & 0.279 \\
\hline Cancer & $226(26.4)$ & $150(30.1)$ & $58(24.4)$ & $18(14.9)$ & 0.002 \\
\hline Diabetes & $250(29.2)$ & 89 (17.9) & $70(29.4)$ & $91(75.2)$ & $<0.0001$ \\
\hline Mortality & $39(4.55)$ & $24(4.82)$ & $10(4.20)$ & $5(4.13)$ & 0.906 \\
\hline Length of stay*** & $5.0(3.0,9.0)$ & $5.0(3.0,9.0)$ & $5.0(3.0,9.0)$ & $5(3.0,10.0)$ & 0.736 \\
\hline
\end{tabular}

Notes: *Mean \pm std; **n (\%); ***median (25th percentile, 75th percentile); ****P-values are from Pearson's Chi-square test for categorical measures with the exception of $\mathrm{HIV}$ (which used the alternative exact test) and analysis of variance for age (continuous).

range 3-9 days). Readmission within 30 days occurred in $17 \%$ (Table 2). None of these clinical outcomes differed by glucose category. The length of stay, readmission rate, and inpatient mortality were similar in the three glucose categories.

Multivariable models were built that forced glucose and age group into the model. Covariates included in the model for length of stay were cancer, chronic renal failure, and cirrhosis. Hazard ratios (HRs) for LoS indicated essentially no effect of glucose (HR: 1.03, 95\% CI: 0.86-1.23 for $126-<180$ versus $<126$; HR: 0.99 , 95\% CI: $0.79-1.25$ for $\geq 180$ versus $<126)$ and shorter LoS among those ages 40-64 (versus 65 and older; HR: 1.23; 95\% CI: 1.04-1.44). The $P$-values for these two factors were 0.95 and 0.01 for glucose and age group, respectively. Thus, age group described significant variation in the hospital LoS, but glucose did not in this cohort. Controlling for age, no differences in the risk of 30-day readmission between the various levels of glucose were detected $(P=0.56)$. Controlling for age, no differences in the risk of in-hospital mortality between the various levels of glucose were detected $(P=0.94)$. This suggested that no effect for glucose (consistent with the bivariate results) and no interaction effect for glucose with age on length of stay $(P=0.20), 30$-day readmission $(P=0.50)$, or mortality $(P=0.91)$ were detected.

\section{Discussion}

This retrospective observational study of CAP admission data revealed that the first glucose measure upon admission did

Table 2 Baseline characteristics of patients admitted for pneumonia and discharged from the hospital

\begin{tabular}{|c|c|c|c|c|c|}
\hline & $\begin{array}{l}\text { Subjects in 30-day } \\
\text { readmission }\end{array}$ & Normal & $\begin{array}{l}\text { Mild } \\
\text { hyperglycemia }\end{array}$ & $\begin{array}{l}\text { Uncontrolled } \\
\text { hyperglycemia }\end{array}$ & $\boldsymbol{P}$-value ${ }^{* * *}$ \\
\hline & & $<126$ mg/dl & $|26-<| 80 \mathrm{mg} / \mathrm{dl}$ & $\geq 180 \mathrm{mg} / \mathrm{dl}$ & \\
\hline Total & 797 & 466 & 219 & 112 & \\
\hline \multicolumn{6}{|l|}{ Age } \\
\hline Years* & $63.8 \pm 13.4$ & $63.0 \pm 13.8$ & $64.8 \pm 12.8$ & $64.8 \pm 12.3$ & 0.201 \\
\hline $65+* *$ & $363(45.6)$ & $208(44.6)$ & $102(46.6)$ & $53(47.3)$ & 0.822 \\
\hline Male** & 387 (48.6) & $217(46.6)$ & $112(5 I . I)$ & $58(51.8)$ & 0.408 \\
\hline Heart failure** & $164(20.6)$ & $81(17.4)$ & $55(25.1)$ & $28(25.0)$ & 0.03 \\
\hline Cirrhosis** & $18(2.26)$ & $10(2.15)$ & $3(1.37)$ & $5(4.46)$ & 0.163 \\
\hline Chronic renal failure ${ }^{* *}$ & 147 (18.4) & $77(16.5)$ & $45(20.6)$ & $25(22.3)$ & 0.234 \\
\hline HIV** & $4(0.5)$ & $4(0.86)$ & 0 & 0 & 0.279 \\
\hline Cancer** & 193 (24.2) & 132 (28.3) & $46(21.0)$ & $15(13.4)$ & 0.002 \\
\hline Diabetes** & $232(29.1)$ & $82(17.6)$ & $64(29.2)$ & $86(76.8)$ & $<0.0001$ \\
\hline 30 day readmission rate** & I 37 (I7.2) & $78(16.7)$ & $36(16.4)$ & $23(20.5)$ & 0.596 \\
\hline
\end{tabular}

Notes: *Mean \pm std; **n (\%); ***P-values are from Pearson's Chi-square test for categorical measures (with the exceptions of cirrhosis and HIV, which used the alternative exact tests) and analysis of variance for age (continuous). 
not predict hospital mortality, length of stay, or readmission for community-acquired pneumonia within 30 days in the University of Kansas Medical Center in the USA.

However, there are several limitations to consider. We were not able to follow these patients after discharge from the hospital except to observe readmissions to the same hospital. Mortality during the hospital stay was uncommon, and we were unable to capture post-discharge mortality. Thus, this study sample may be too small to capture possible differences in longer term mortality outcomes across different levels of admission glucose. We were not able to examine changes in serum glucose throughout the hospital stay. There was no structured protocol in treatment for patients with increase serum glucose, which was instead left to the discretion of the health care providers within our center. A single measure of hyperglycemia at admission might reflect the production of stress related counter-regulatory hormones due to acute illness. We are unable to exclude the possibility that severity of illness was differentially distributed across levels of glucose control. This question should be tested in a larger study sample in which measures of severity of illness are available.

A US study reported by Labraere et $\mathrm{al}^{6}$ reached similar conclusions to our own. In contrast, our findings differ from a recently published German prospective study by Lepper et $\mathrm{al}^{7}$ showing that serum glucose at the time of hospital admission for community-acquired pneumonia $(n=6870)$ was a predictor of death at 90 days after admission. This prospective study had a longer follow up time period, and captured specific patient-level clinical factors such as confusion, respiratory rate, and blood pressure. Our study population was demographically different and had a threefold higher rate of malignancy than that in Lepper's trial. ${ }^{7}$ The underlying difference in study population may account in part for disparate conclusions.

\section{Conclusion}

Our retrospective observational study of community-acquired pneumonia admission data revealed that first glucose measure upon admission did not predict hospital mortality, length of stay, or readmission for community-acquired pneumonia within 30 days. Further research on the impact of hyperglycemia needs to be done in larger, diverse samples of CAP patients.

\section{Author contributions}

All authors contributed equally to the conception, design and the drafting of the manuscript. Jonathan D Mahnken was responsible for the statistical analysis. All authors read and approved the final manuscript.

\section{Disclosure}

Rajib K Bhattacharya is a promotional speaker and involved in an industry sponsored clinical trial for Sanofi Aventis. Sally K Rigler and Jonathan D Mahnken have no competing interests to declare.

\section{References}

1. Valdez R, Narayan KM, Geiss LS, Engelgau MM. Impact of diabetes mellitus on mortality associated with pneumonia and influenza among non-Hispanic black and white US adults. Am J Public Health. 1999; 89(11):1715-1721.

2. Moss SE, Klein R, Klein BE. Cause-specific mortality in a population-based study of diabetes. Am Journal Public Health. 1991;81(9):1158-1162.

3. Fry AM, Shay DK, Holman RC, Curns AT, Anderson LJ. Trends in hospitalizations for pneumonia among persons aged 65 years or older in the United States, 1988-2002. JAMA. 2005;294(21):2712-2719.

4. Frei CR, Mortensen EM, Copeland LA, et al. Disparities of care for African-Americans and Caucasians with community-acquired pneumonia: a retrospective cohort study. BMC Health Services Research. 2010;10:143.

5. Murad MH, Coburn JA, Coto-Yglesias F, et al. Glycemic control in noncritically ill hospitalized patients: a systematic review and meta-analysis. J Clin Endocrinol Metab. 2012;97(1):49-58.

6. Labarere J, Stone RA, Scott Obrosky D, et al. Factors associated with the hospitalization of low-risk patients with community-acquired pneumonia in a cluster-randomized trial. J Gen Intern Med. 2006;21(7):745-752.

7. Lepper PM, Ott S, Nuesch E, et al. Serum glucose levels for predicting death in patients admitted to hospital for community acquired pneumonia: prospective cohort study. BMJ. 2012;344:e3397.

8. Castellanos MR, Szerszen A, Saifan C, et al. Fasting hyperglycemia upon hospital admission is associated with higher pneumonia complication rates among the elderly. Int Arch Med. 2010;3:16.
International Journal of General Medicine

\section{Publish your work in this journal}

The International Journal of General Medicine is an international, peer-reviewed open-access journal that focuses on general and internal medicine, pathogenesis, epidemiology, diagnosis, monitoring and treatment protocols. The journal is characterized by the rapid reporting of reviews, original research and clinical studies across all disease areas.

\section{Dovepress}

A key focus is the elucidation of disease processes and management protocols resulting in improved outcomes for the patient.The manuscript management system is completely online and includes a very quick and fair peer-review system. Visit http://www.dovepress.com/ testimonials.php to read real quotes from published authors. 